\title{
RESSECÇÃO LAPAROSCÓPICA DE TUMORES BENIGNOS DO FÍGADO: POSIÇÃO ATUAL
}

\author{
LAPAROSCOPIC LIVER RESECTION FOR BENIGN TUMORS: THE CURRENT POSITION \\ Paulo HERMAN ${ }^{1}{ }^{\odot}$, Gilton Marques FONSECA ${ }^{1 \oplus}$, Jaime Arthur Pirola KRUGER $^{1}{ }^{\oplus}$, Vagner Birk JEISMANN ${ }^{\oplus}$, \\ Fabricio Ferreira COELHO ${ }^{1}$
}

RESUMO - RACIONAL: As principais indicações das hepatectomias video-laparoscópicas (HVL), inicialmente, eram nas lesões hepáticas benignas. À medida que a HVL se tornou mais popular, as indicações de doenças malignas superaram as de doenças benignas. Este estudo teve como objetivo discutir as indicações e resultados da HVL para o tratamento de tumores hepáticos benignos. MÉTODOS: De 445 HVL realizadas em um único centro, 100 (22,4\%) foram para tumores benignos. Os autores discutem as indicações para ressecção e apresentam seus resultados perioperatórios. RESULTADOS: No total, 100 pacientes com tumores benignos foram avaliados, a saber: 66 casos de adenomas hepatocelulares; 14 de neoplasia mucinosa biliar; 13 de hiperplasia nodular focal; 4 de angiomiolipomas; e 3 de hemangiomas. O tamanho médio das lesões foi de 7,6 cm $(3,1 \mathrm{a} 19,6 \mathrm{~cm})$ A taxa de morbidade total foi de 19\%, sendo $9 \%$ classificados como Clavien-Dindo 3 ou 4 e não foi observada mortalidade. CONCLUSÃO: A HVL para tumores hepáticos benignos é segura e apresenta excelentes resultados. No entanto, as indicações para cirurgia são cada vez mais restritas, não sendo recomendável indicar a ressecção somente por se tratar de procedimento minimamente invasivo.

DESCRITORES: Laparoscopia. Adenoma de células hepáticas. Cistadenoma. Hiperplasia nodular focal do Fígado. Angiomiolipoma. Hemangioma.

ABSTRACT - BACKGROUND: The main indications of the use of laparoscopic liver surgery (LLS), in the early days, were benign liver lesions. As LLS became more popular, indications for malignant diseases outnumbered those for benign ones. This study aims to rule out the indications and results of LLS for the treatment of benign liver tumors. METHODS: Out of 445 LLS performed in a single center, 100 (22.4\%) were for benign tumors. The authors discuss the indications for resection and present their perioperative results. RESULTS: In total, 100 patients with benign tumors were evaluated. Specifically, these were as follows: 66 cases of hepatocellular adenomas; 14 cases of biliary mucinous neoplasm 13 cases of focal nodular hyperplasia; 4 cases of angiomyolipomas; and 3 cases of hemangiomas with a mean size of $7.6 \mathrm{~cm}$ (ranging from 3.1 to $19.6 \mathrm{~cm}$ ). The total morbidity rate was $19 \%$, with $9 \%$ classified as Clavien-Dindo grades 3 or 4 . No mortality was observed. CONCLUSION: LLS for benign liver tumors is safe and presents excellent results. However, indications for resection are increasingly restricted and should not be performed just because it is a minimally invasive procedure.

HEADINGS: Laparoscopy. Adenoma, Liver Cell. Cystadenoma. Focal nodular hyperplasia. Angiomyolipoma. Hemangioma.

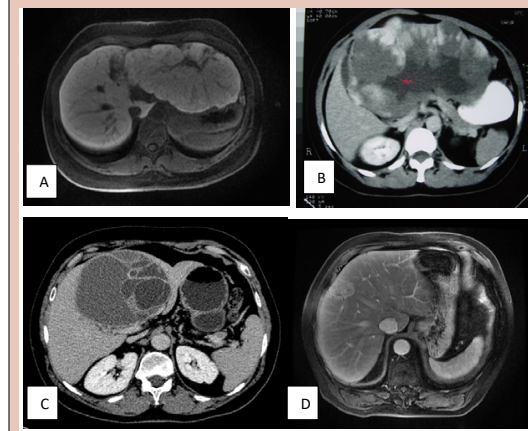

Figura 1 - Achados radiológicos típicos para o diagnóstico de: hiperplasia nodular focal sintomática no lobo esquerdo comprimindo - estômago (A); hemangioma hepático sintomático no lobo esquerda comprimindo o estômago (B); neoplasia cística biliar mucinosa no segmento 4 do fígado (C); e angiomiolipoma entre os segmentos 4 e 8 (D).

\section{Mensagem central}

Apesar dos excelentes resultados, as indicações para ressecção laparoscópica devem ser as mesmas da cirurgia aberta e que o leque de indicações não deve ser ampliado apenas por se tratar de um procedimento minimamente invasivo.

\section{Perspectivas}

A cirurgia laparoscópica do fígado para tumores hepáticos benignos é viável e segura, mesmo quando grandes ressecções são necessárias. Cada tumor hepático benigno tem indicação específica e restritiva para ressecção.

Trabalho realizado na ${ }^{1}$ Unidade de Cirurgia Hepática, Hospital de Clínicas, Departmento of Gastroenterologia, Faculdade de Medicina da Universidade of São Paulo- USP - Sao Paulo, Brasil.

Como citar esse artigo: Herman P, Fonseca GM, Jaime Arthur Pirola Kruger JAP, Jeismann VB, Coelho FF. Ressecção laparoscópica de tumores benignos do fígado: Posição atual. ABCD Arq Bras Cir Dig. 2021;34(4):e1641. https://doi.org/10.1590/0102-672020210002e1641

Correspondência:

Paulo Herman

Email: pherman@uol.com.br
Fonte de financiamento: Não.

Conflito de interesses: Não.

Recebido: 22/10/2021

Aceito: 25/11/2021 
INTRODUÇÃO

A hepatectomia video-laparoscópica (HVL) vem ganhando ampla aceitação em todo o mundo, sendo cada vez mais empregada para o tratamento de doenças hepáticas benignas e malignas ${ }^{8,13}$. Com os avanços técnicos e a maior experiência, os resultados são comparáveis ou até melhores do que os da cirurgia aberta, resultando no reconhecimento e aceitação da abordagem minimamente invasiva para ressecções hepáticas. No entanto, é recomendado que a cirurgia laparoscópica do fígado seja realizada por um cirurgião com experiência em cirurgia hepática e com treinamento em laparoscopia avançada, possibilitando em uma menor curva de aprendizado. No início da HVL, os melhores candidatos eram pacientes com lesões periféricas localizadas nos segmentos lateral e anterior esquerdos, exigindo ressecções limitadas ${ }^{23}$. Mais recentemente, no entanto, um número crescente de ressecções hepáticas maiores e complexas tem se mostrado factíveis e realizadas com segurança, quando em centros especializados ${ }^{25,10}$. Hoje em dia, mesmo ressecções extensas ou transplantes com doadores vivos do lobo direito são realizados rotineiramente por grupos de especialistas com excelentes resultados. As vantagens da HVL incluem menores taxas de sangramento, menos dor pósoperatória, menos complicações pulmonares, menor tempo de recuperação e internação hospitalar e, menores taxas de hérnia incisional ${ }^{6}$. Como a abordagem laparoscópica oferece excelentes resultados cirúrgicos, além de melhores resultados estéticos e um rápido retorno às atividades diárias, tornou-se amplamente aceito para o tratamento de doenças hepáticas benignas ${ }^{14,30}$.

A maioria das ressecções laparoscópicas hepáticas em seus primeiros anos, foram para doenças benignas. De fato, no primeiro consenso internacional em Louisville (2008), mais da metade de todas HVL realizadas até então eram para tumores benignos. Naquela época, a maioria das cirurgias (20\%) era para cistos hepáticos (não necessariamente ressecções hepáticas) ou para indicações discutíveis, como hiperplasia nodular focal ou hemangiomas (36\% dos casos) ${ }^{24}$. Apesar da ressecção laparoscópica apresentar vantagens por se tratar de procedimento minimamente invasivo, atendendo ao desejo do cirurgião de realizar uma ressecção fácil e possibilitando uma aceitação mais fácil do paciente como um procedimento menos invasivo, deve-se enfatizar que as indicações cirúrgicas devem ser as mesmas dos procedimentos abertos. Como a experiência com HVL cresceu na maioria dos grupos dedicados à cirurgia hepatobiliar e um número maior de pacientes com doenças malignas foi operado, as indicações para tumores benignos tornaram-se menos frequentes e mais baseadas em evidências. Em 2016, Ciria et al. relataram que 35\% de todos os casos de HVL em todo o mundo foram por doenças benignas ${ }^{6}$. Da mesma forma, uma pesquisa recente incluindo 2.887 pacientes mostrou que $43 \%$ dos pacientes foram operados devido a doenças benignas do fígado ${ }^{25}$.

O objetivo do presente estudo foi apresentar nossa experiência com a ressecção laparoscópica de tumores hepáticos benignos e discutir as indicações e resultados do ponto de vista acadêmico. Os autores procuraram apontar as indicações atuais para ressecção em um contexto baseado em evidências.

\section{MÉTODOS}

Todos os casos de HVL de uma única instituição de referência foram revisados de um banco de dados prospectivo (Redcap). As ressecções para tumores benignos do fígado entre 2005 e 2021 foram revisadas. Idade, sexo, tamanho dos nódulos, indicação de tratamento cirúrgico, tipo de ressecção hepática (menor quando foram ressecados até dois segmentos), necessidade de transfusão, morbidade (de acordo com a classificação de Dindo-Clavien) ${ }^{9}$, tempo de internação e mortalidade foram avaliados. Dados sobre variáveis contínuas foram coletados e definidos como valores mínimo, máximo e médio. O diagnóstico pré-operatório foi baseado em tomografia computadorizada (TC) ou ressonância magnética (RM) e, em sete casos, foi necessária biópsia. O diagnóstico foi confirmado por meio do exame histológico da amostra.

\section{RESULTADOS}

Dentre $445 \mathrm{HVL}, 100$ (22,4\%) foram para tumores benignos, 66 (66\%) foram para adenoma hepático (AH), 14 (14\%) foram para neoplasia cística mucinosa biliar (NCMB), 13 (13\%) foram para hiperplasia nodular focal (HNF), 4 (4\%) foram para angiomiolipoma (AML) (Figura 1) e 3 (3\%) foram para hemangiomas hepáticos $(\mathrm{HH})$. Na Tabela 1, apresentamos os dados das HVL para diferentes tumores hepáticos benignos nos quais a ressecção laparoscópica foi realizada.

Oitenta e seis pacientes eram do sexo feminino e 14 do masculino, com média de idade de 38,6 anos (11-77 anos). O tamanho médio dos adenomas foi de $7,0 \mathrm{~cm}$; das neoplasias biliares mucinosas císticas, $7,8 \mathrm{~cm}$; das hiperplasias nodulares focais, 7,9 cm; dos angiomiolipomas, 5,8 cm; e dos hemangiomas hepáticos, 7,6 cm. Em 22,4\% dos casos, foram realizadas HVL maiores. A perda sanguínea estimada foi de $245 \mathrm{ml}(10 \mathrm{ml}-1500 \mathrm{ml})$, e a taxa de transfusão foi de $6 \%$. A morbidade da série foi de 19,3\%, mas apenas 9,1\% apresentaram complicações classificadas como Dindo-Clavien 3 ou 4.

As complicações mais comuns foram pneumonia (três casos), abscesso intra-abdominal (dois casos) e fístulas biliares (dois casos). O tempo médio de internação foi de 4,6 dias. Nenhuma mortalidade foi observada. Erro diagnóstico pré-operatório foi observado em nove casos, todos os quais foram confirmados histologicamente no pós-operatório como hiperplasia nodular focal. Nestes casos, as hipóteses pré-operatórias para ressecção foram adenoma hepático em oito casos e carcinoma hepatocelular em um.
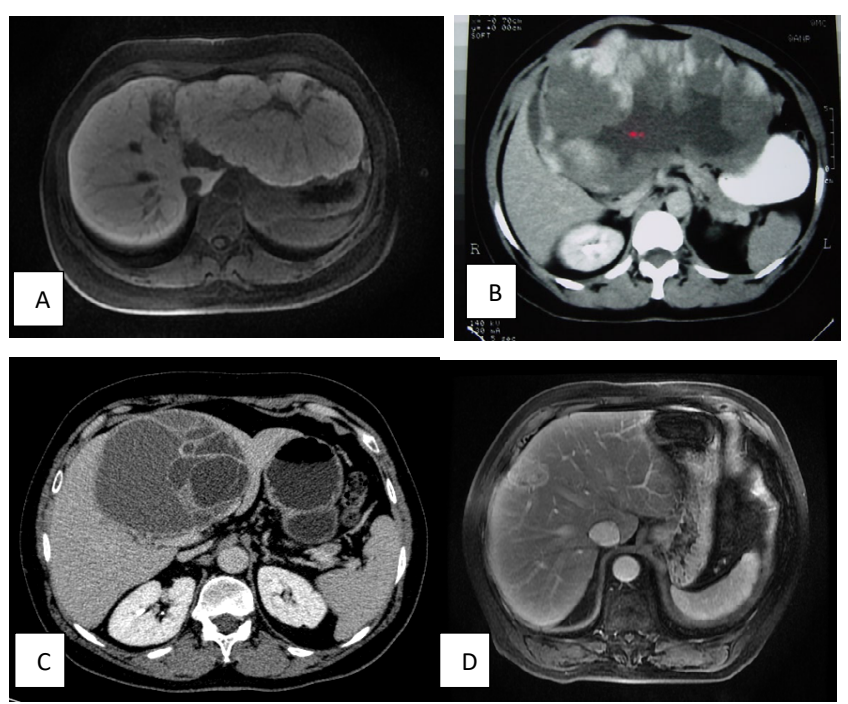

Figura 1 - Achados radiológicos típicos para o diagnóstico de: hiperplasia nodular focal sintomática no lobo esquerdo comprimindo o estômago (A); hemangioma hepático sintomático no lobo esquerda comprimindo o estômago (B); neoplasia cística biliar mucinosa no segmento 4 do fígado (C); e angiomiolipoma entre os segmentos 4 e 8 (D). 
Tabela 1 - Dados dos pacientes submetidos à ressecção laparoscópica de tumores hepáticos benignos

\begin{tabular}{|c|c|c|c|c|c|c|}
\hline & & $\begin{array}{l}\text { Adenoma } \\
\text { Hepático }\end{array}$ & $\begin{array}{l}\text { Neoplasia } \\
\text { cística biliar } \\
\text { mucinosa }\end{array}$ & $\begin{array}{l}\text { Hiperplasia } \\
\text { nodular focal }\end{array}$ & $\begin{array}{c}\text { Hepatic } \\
\text { hemangioma }\end{array}$ & $\begin{array}{c}\text { Hepatic } \\
\text { angiomyolipoma }\end{array}$ \\
\hline Número de pacientes (\%) & & $66(66 \%)$ & $14(14 \%)$ & $13(13 \%)$ & $3(3 \%)$ & $4(4 \%)$ \\
\hline \multirow{2}{*}{ Sexo } & Masculino & $9(13.6)$ & $2(14.3)$ & $1(7,7)$ & $1(33.3)$ & $1(25)$ \\
\hline & Feminino & $57(86.4$ & $12(85.7)$ & $12(92.3)$ & $2(66.7)$ & $3(75)$ \\
\hline Idade (anos) & Min - Max (Média) & $11-70(35.0)$ & $28-75(52.5)$ & $16-41(30.6)$ & $40-69(56.0)$ & $43-77(61.75)$ \\
\hline Tamanho do tumor (cm) & Min - Max (Média) & $3.1-19.6(7.0)$ & $4.5-12.5(7.8)$ & $4.4-14.0(7.9)$ & $5.0-11.1(7.6)$ & $5.1-6.4(5.8)$ \\
\hline Tempo de internação (dias) & Min - Max (Média) & $2-29(4.8)$ & $1-7(4.6)$ & $2-9(3.8)$ & $1-3(2)$ & $4-18(8.5)$ \\
\hline \multirow{2}{*}{ Extensão da ressecção } & Maior & $12(18.2)$ & $8(57.1)$ & $1(7.7)$ & $0(0)$ & $1(25)$ \\
\hline & Menor & $54(81.8)$ & $6(42.9)$ & $12(92.3)$ & $3(100)$ & $3(75)$ \\
\hline Tempo operatório & Min - Max (Média) & $83-495(253)$ & $120-505$ (297) & $80-400(201)$ & $125-210(157)$ & $120-600(360)$ \\
\hline $\begin{array}{l}\text { Perda estimada de } \\
\text { sangue }(\mathrm{mL})\end{array}$ & Min - Max (Média) & $10-1500(231)$ & $15-1000(265)$ & $10-500(144)$ & $10-20(15)$ & $50-1500(650)$ \\
\hline \multirow{2}{*}{ Transfusão } & Sim & $5(7.5)$ & $0(0)$ & $0(0)$ & $0(0)$ & $1(75)$ \\
\hline & Não & $61(92.5)$ & $14(100)$ & $13(100)$ & $3(100)$ & $3(25)$ \\
\hline \multirow{3}{*}{ Morbidade perioperatória } & Não & $52(78.8)$ & $13(92.9)$ & $11(84.6)$ & $3(100)$ & $2(50)$ \\
\hline & Clavien I/II & 7 (10.6) & $1(7.1)$ & $2(15.4)$ & $0(0)$ & $0(0)$ \\
\hline & Clavien III/IV & $7(10.6)$ & $0(0)$ & $0(0)$ & $0(0)$ & $2(50)$ \\
\hline
\end{tabular}

\section{DISCUSSÃO}

A melhora dos métodos diagnósticos durante os últimos anos levaram a um aumento na detecção de tumores hepáticos benignos. Além disso, diagnósticos mais precisos tornaram-se possíveis, permitindo aos cirurgiões hepáticos compreender o comportamento das lesões benignas. O aumento do diagnóstico, associado ao entusiasmo pela técnica minimamente invasiva, levou ao aumento das indicações cirúrgicas. Toro et al. demonstraram que a introdução da abordagem laparoscópica resultou em aumento da taxa de ressecções hepáticas para tumores benignos, mesmo naqueles com indicação duvidosa ${ }^{28}$. As indicações para ressecção de tumores hepáticos benignos são: 1) doenças sintomáticas que impactam na qualidade de vida; e / ou 2) risco de complicações, como transformação maligna ou ruptura. As indicações mais frequentes para HVL são adenoma de células hepáticas e neoplasia biliar cística mucinosa pelo risco de complicações.

As indicações para ressecção de hemangiomas ou hiperplasia nodular focal são casos anedóticos em que sintomas significativos estão presentes. Aqui, discutiremos cada um desses tumores, enfocando as indicações clínicas para ressecção.

É consenso que as indicações para ressecção laparoscópica de tumores hepáticos benignos, apesar de o procedimento ser factível e seguro, não devem ser ampliadas diante da adoção de abordagem menos invasiva ${ }^{10,23,24}$. Baixas taxas de morbidade ( $<15-20 \%$ ) e nenhuma mortalidade ${ }^{13,14,30}$ são as metas pós-HVL

Em nossa experiência com 100 ressecções laparoscópicas para tumores hepáticos benignos, a principal indicação foi $\mathrm{AH}(\mathrm{n}=66 ; 66 \%)$. Em toda a série, ocorreram $22(22 \%)$ ressecções hepáticas maiores, e o tamanho médio das lesões foi de $7,6 \mathrm{~cm}$. A perda sanguínea estimada foi de $245 \mathrm{ml}, 6,0 \%$ dos pacientes receberam transfusão de sangue e não foram necessárias conversões para cirurgia aberta. Complicações pós-operatórias significativas (Dindo-Clavien $\geq 3$ ) foram observadas em $9,0 \%$ dos pacientes, sem mortalidade operatória. O tempo médio de internação foi de 4,6 dias. A seguir, discutiremos cada uma das indicações para a ressecção de tumores hepáticos benignos.

\section{Adenoma hepático}

$\mathrm{O}$ AH é um tumor hepático benigno raro que acomete mulheres jovens em idade reprodutiva, com incidência crescente devido ao uso disseminado de contraceptivos orais (ACO) 3,15,20. $\mathrm{O}$ HA é mais frequentemente assintomático e frequentemente encontrado incidentalmente durante exames de imagem para causas não relacionadas. A incidência estimada de HA é de um por milhão de habitantes, aumentando para 30-40 por milhão em usuários de contraceptivos orais de longo prazo ${ }^{20}$. A associação com doenças de armazenamento de glicogênio tipos 1 e 3, o uso de esteróides anabolizantes, obesidade e síndromes metabólicas é também bem estabelecido ${ }^{20}$.

Com base nas características genéticas e fenotípicas, quatro subgrupos $\mathrm{AH}$ foram inicialmente relatados: (a) $\mathrm{AH}$ com mutação da HNF1 $\alpha$, representando $30-40 \%$ dos casos de adenoma, caracterizado por mutações inativadoras no gene fator nuclear hepatocitário 1-alfa (HNF1A) e leva a um nódulo de fenótipo gorduroso e expressão negativa de proteína de ligação de ácido graxo hepático, também conhecido como "adenoma esteatótico"; (b) AH inflamatório (I-AH), representando $40-50 \%$ de todos os adenomas, tem características inflamatórias relacionadas à ativação da via JAK-STAT morfologicamente caracterizado por telangiectasias, inflamação, reação ductular e coloração para proteína $C$ reativa; (c) $\mathrm{AH}$ mutado com $\beta$-catenina (b-AH) representando $10 \%$ dos adenomas, tem morfologias variáveis, coloração positiva com glutamina sintetase e expressão nuclear de $\beta$-catenina; e (d) aproximadamente $10 \%$ dos adenomas permaneceram sem classificação, sem características histológicas características ${ }^{21}$.

Mais recentemente, uma nova classificação molecular de $\mathrm{AH}$ foi proposta com base no perfil molecular em quatro vias diferentes: ativação de $\beta$-catenina, interleucina 6 / JAK / STAT e via sonic hedgehog sônica e inativação de HNF1A. Com base nessas características moleculares, um novo subtipo foi identificado (o sonic hedgehog $\mathrm{AH}$ [shAH), e os b-AHs foram divididos em dois subgrupos adicionais de acordo com a presença de mutações de CTNNB1 (exon 3 vs. exons 7/8). De acordo com essa nova classificação, a presença de mutações CTNNB1 no exon 3 está associada a um alto risco de transformação maligna. Além disso, o shAH foi associado a um alto risco de ruptura e sangramento. É importante notar que alguns tumores apresentam características mistas comuns aos subtipos inflamatórios e ativados por $\beta$-catenina (mistos bexon7 / 8I-AH e bexon3I-AH). Nessa nova classificação, 7\% dos adenomas ainda permanecem sem classificação ${ }^{21,22}$

A nova classificação com oito subtipos de $\mathrm{AH}$ mostra a complexidade e heterogeneidade da doença. No entanto, do ponto de vista prático, não é útil, pois é necessário um processo molecular complexo para identificar todos os subtipos. Além disso, toda a amostra (peça cirúrgica) do adenoma é necessária para uma avaliação precisa. Por esses motivos, essa classificação ainda não é totalmente aplicável na prática diária ${ }^{20}$. 
O novo conhecimento do perfil molecular e seu impacto no comportamento clínico levou à mudanças no diagnóstico e tratamento desta doença desafiadora. Na prática diária, o diagnóstico de subtipos de $\mathrm{AH}$ é baseado em achados de ressonância magnética, cuja capacidade de distinguir diferentes subtipos de adenomas (especialmente $\mathrm{AH}$ esteatótico e inflamatório) está bem estabelecida ${ }^{29}$. Em caso de dúvida, pode-se fazer uma biópsia percutânea.

As mudanças na estratégia de tratamento são contínuas, com base no risco de complicações de acordo com os subtipos de adenoma. Até recentemente, em mulheres com adenomas medindo $5,0 \mathrm{~cm}$ ou mais, a ressecção era indicada devido ao risco estimado de $5 \%$ a $8 \%$ para transformação maligna ou $21 \%$ a $29 \%$ para ruptura e hemorragia. Nault et al. relataram uma grande série com 411 pacientes, mostrando que sangramento sintomático ocorreu em $14 \%$ dos pacientes e que $3 \%$ dos pacientes apresentavam degeneração maligna de $\mathrm{AH}$ para carcinoma hepatocelular ${ }^{23}$. Farges et al. reportaram que a prevalência de malignidade em pacientes com $\mathrm{AH}$ era 10 vezes maior em homens do que em mulheres, sugerindo a ressecção rotineira de todos os adenomas em homens, independentemente do tamanho da lesão ${ }^{12}$. Atualmente, a ressecção deve ser recomendada para todos os adenomas que afetam homens e adenomas não esteatóticos maiores que $5 \mathrm{~cm}$, após 6 a 12 meses de interrupção do anticoncepcional oral e perda de peso, em mulheres ${ }^{14}$.

Quando o tratamento cirúrgico é indicado, excelentes resultados foram observados, mesmo quando grandes ressecções foram necessárias ${ }^{20}$. Já demonstramos anteriormente resultados excelentes após o tratamento laparoscópico de $\mathrm{AHs}$, com baixo índice de complicações e sem mortalidade ${ }^{13}$. Pacientes com $\mathrm{AH}$ parecem ser especialmente beneficiados pela abordagem laparoscópica, em particular, pacientes jovens do sexo feminino, onde uma abordagem menos invasiva oferece melhores resultados pós-operatórios e excelentes resultados cosméticos. A abordagem laparoscópica deve ser considerada o padrão ouro para pacientes com adenomas de células hepáticas quando realizada por especialistas ${ }^{13,14}$. Em nossa experiência, 66 pacientes com $\mathrm{AH}$ foram submetidos à ressecção laparoscópica, incluindo $12(18,2 \%)$ ressecções hepáticas maiores, com baixas taxas de morbidade $(10,6 \%$ classificados como Dindo-Clavien graus 3 ou 4) e sem mortalidade. A abordagem convencional (aberta) foi empregada para a ressecção de lesões de localização central ou em pacientes com tumores muito grandes $(>10 \mathrm{~cm})$.

\section{Neoplasia cística mucinosa biliar}

A NCMB é um cisto neoplásico raro, originalmente denominado cistoadenoma biliar. Esses tumores císticos prémalignos são frequentemente detectados incidentalmente em exames radiológicos para outras causas ${ }^{27}$. Essas lesões se originam do epitélio biliar e representam menos de $1 \%$ de todas as lesões císticas do fígado ${ }^{11}$. Edmondson descreveu o NCMB como uma lesão cística multilocular revestida por epitélio colunar com um acompanhando denso estroma celular "semelhante ao ovário" 1.

As NCMB são normalmente detectadas em mulheres jovens (> 90\%) entre 30 e 50 anos de idade sendo raramente observados em homens. Geralmente são cistos grandes e complexos, muitas vezes localizados no lobo esquerdo do fígado com múltiplas septações, e projeções papilares podem ser vistas originando-se dos septos ou da parede do cisto. A frequência de degeneração maligna foi relatada como variando entre $20 \%$ e $30 \%$ dos casos ${ }^{1}$. Por esse motivo, a ressecção é o tratamento de escolha, oferecendo a melhor chance de cura, e ambas as abordagens aberta e minimamente invasiva podem ser empregadas, dependendo do tamanho, localização da lesão e proximidade com estruturas biliares e vasculares. A enucleação dessas lesões pode ser um procedimento muito complexo, uma vez que os NCMB estão situados muito próximos ao ducto biliar (ducto esquerdo ou confluência biliar) e, muitas vezes, a hepatectomia esquerda é o procedimento de escolha.

Na maior série até o momento, relatando 221 casos, a sobrevida livre de recorrência em cinco anos foi de $61,4 \%$, e as recorrências tendiam a ocorrer localmente, muitas vezes causadas por uma ressecção inicial incompleta ${ }^{18}$. Em nossa série, tratamos 14 pacientes com $\mathrm{NCMB}$, todos grandes (tamanho médio $=7,8$ $\mathrm{cm}$; variação $=4,5-12,5 \mathrm{~cm}$ ) e situados no lobo esquerdo do fígado. Oito hepatectomias esquerdas e seis cistectomias foram realizadas sem morbidade significativa (Dindo-Clavien grau 3 ou $4=0$ ) e, sem mortalidade. Nenhuma recidiva foi observada.

\section{Hiperplasia nodular focal}

A HNF é o segundo tumor benigno mais comum do fígado, geralmente diagnosticada incidentalmente durante uma ultrassonografia abdominal. Geralmente afeta mulheres entre 30 e 50 anos e sua incidência não é influenciada pelo uso de contraceptivos orais. É caracterizada pela presença de uma massa hipervascular bem delimitada com septos fibrosos e uma cicatriz estrelada central sem realce. A HNF representa uma resposta hiperplásica a uma malformação arteriovenosa, sendo mais comum em mulheres jovens, embora até $10 \%$ dos casos possam ocorrer em homens ${ }^{15}$. Apresenta curso clínico benigno, sendo a maioria assintomática; desconforto abdominal leve é raramente observado. Uma vez que FNH raramente apresenta complicações, como risco de ruptura ou degeneração maligna, tratamento raramente é necessário ${ }^{17}$.

Existem raras situações em que a ressecção pode ser indicada: uma grande massa no lado esquerdo levando à compressão gástrica sintomática ou uma lesão de crescimento rápido. Em nossa experiência, ressecamos $13 \mathrm{HNFs}$, sendo 8 diagnosticados incorretamente como adenomas hepáticos e 1 como carcinoma hepatocelular (9 erros diagnósticos); um paciente foi operado devido ao rápido crescimento do tumor e outros três devido a um grande tumor no segmento lateral esquerdo levando a sintomas de compressão gástrica (Figura 1).

\section{Hemangioma hepático}

$\mathrm{O} \mathrm{HH}$ é o tumor benigno mais comum do fígado, de etiologia desconhecida e com preponderância feminina com proporção mulher-homem de 15:1. Esta malformação vascular congênita composta por um emaranhado de vasos sanguíneos pode apresentar receptores estrogênicos.

A maioria dos pacientes com HH é assintomática e o hemangioma geralmente é um achado incidental na avaliação radiológica não relacionada. Grandes hemangiomas podem eventualmente causar dor abdominal devido à trombose intratumoral. A ressecção do hemangioma raramenteé necessária, exceto nas seguintes circunstâncias: sintomas com uma resposta pobre aos analgésicos, impacto na qualidade de vida do paciente, síndrome de Kasabach-Merrit e um grande tumor do lado esquerdo com compressão gástrica sintomática. As complicações, incluindo hemorragia ou ruptura, são extremamente raras. A rara síndrome de Kasabach-Merritt ocorre em menos de 3\% dos pacientes e é causada pelo aprisionamento de plaquetas dentro do tumor, levando à ativação da cascata de coagulação, resultando em trombocitopenia e fibrinólise.

Umagrande série de nosso grupo avaliou retrospectivamente 249 pacientes com hemangiomas, dos quais $27,3 \%$ eram maiores que $4 \mathrm{~cm}$ e $6,4 \%$ maiores que $10 \mathrm{~cm}$. Trinta por cento eram sintomáticos; entretanto, apenas oito pacientes $(3,2 \%)$ foram submetidos a tratamento cirúrgico ${ }^{16}$.

Em nossa série, a abordagem conservadora não cirúrgica sempre foi adotada, visto que as complicações de impacto clínico são extremamente raras. A ressecção deve ser evitada mesmo na presença de dor, pois a ressecção hepática apresenta maior morbidade quando comparada ao curso natural da doença. Outras 
etiologias para a dor devem ser investigadas (síndrome dispéptica, doenças biliares) e, se for determinado que a causa da dor é o $\mathrm{HH}$, ela deve ser controlada com analgésicos. A enucleação do hemangioma quando indicada a cirurgia é viável, porém, em nossa experiência, leva a mais sangramento; portanto, preferimos seccionar o tecido hepático normal próximo ao tumor para preservar o parênquima. Atenção especial deve ser dada aos pacientes que apresentam hemangiomas maiores que $10 \mathrm{~cm}$; nesses casos, a dor de forte intensidade é mais prevalente (37,5\%), mas o tamanho da lesão por si só não deve ser uma indicação para ressecção. Nesses grandes tumores, a ressecção laparoscópica é bastante complicada e, às vezes, impossível devido à difícil mobilização do fígado. Em nossa experiência, o tamanho médio dos $\mathrm{HH}$ s ressecados foi de $7,6 \mathrm{~cm}$, e as principais indicações foram dor abdominal (um caso) e grandes tumores do segmento lateral esquerdo levando à compressão gástrica (dois casos) (Figura 1). Nos poucos casos em que a ressecção foi realizada, não houve morbidade ou mortalidade.

\section{Angiomiolipoma}

O AML é um tumor mesenquimal sólido raro, acometendo geralmente os rins, fazendo parte do grupo dos tumores de células epitelióides perivasculares. O AML hepático é raro, com aproximadamente 600 casos relatados ${ }^{19}$. Na avaliação por TC ou RM contrastada, aparece como um tumor hipervascular com uma fase de lavagem rápida ("washout") mimetizando carcinoma hepatocelular em parênquima hepático normal ${ }^{26}$, representando um desafio diagnóstico, especialmente quando o conteúdo de gordura na avaliação radiológica, é baixo (Figura 1).

O AML hepático geralmente apresenta curso benigno. No entanto, um comportamento agressivo com doença recorrente ou metástase pode ser visto, embora não haja dados no momento para prever o curso natural deste tumor. Devido ao seu difícil diagnóstico e eventual comportamento potencialmente agressivo, uma biópsia para confirmação do diagnóstico e avaliação da celularidade pode ser necessária ${ }^{19}$.

Os critérios para avaliar o comportamento do AML foram recentemente propostos 4 :

- Benigno (sem características preocupantes): tamanho do tumor $<5 \mathrm{~cm}$, bem delimitado, sem infiltração, baixo grau nuclear e celularidade, atividade mitótica $\leq 1$ / 50 HPF, sem invasão vascular.

Potencial de malignidade incerto: pleomorfismo / apenas células gigantes multinucleadas, ou tamanho $>5 \mathrm{~cm}$.

- Comportamento agressivo: duas ou mais das seguintes características preocupantes: tamanho $>5 \mathrm{~cm}$, infiltração periférica, alto grau nuclear e celularidade, atividade mitótica> 1/50 HPF e invasão vascular.

De acordo com a classificação de tumores da Organização Mundial de Saúde: os principais preditores de risco de doença mais agressiva e comportamento metastático são atipia nuclear significativa, pleomorfismo difuso e atividade mitótica $>1$ mitose por $\mathrm{mm}^{2}$. Desta forma, na presença de grandes tumores ou quando a biópsia evidenciar pleomorfismo ou atividade mitótica, a ressecção é recomendada. Finalmente, em nossa série, quatro pacientes com AML hepático foram submetidos à ressecção. Esses casos apresentavam tumores maiores que $5 \mathrm{~cm}$ e foram submetidos à biópsia percutânea para confirmação do diagnóstico. Os pacientes foram submetidos à HVL devido a um potencial de malignidade incerto com excelentes resultados e sem recidiva.

\section{CONCLUSÕES}

As hepatectomias laparoscópicas para tumores hepáticos benignos são factíveis e seguras, mesmo quando grandes ressecções são necessárias. Além disso, menos dor, menor tempo de recuperação e melhor cosmética são a regra. Cada tumor hepático benigno tem indicação específica e restritiva para ressecção. Essas indicações geralmente se devem à presença de sintomas que impactam na qualidade de vida ou ao risco de complicações, como degeneração maligna ou ruptura e sangramento. Antes de se considerar a indicação cirúrgica, recomenda-se a discussão multidisciplinar de um caso, não apenas para confirmar a hipótese diagnóstica ou eventual necessidade de biópsia, mas para estabelecer a melhor abordagem terapêutica.

Os autores reenfatizam que as indicações para ressecção devem ser as mesmas da cirurgia aberta e que o leque de indicações não deve ser ampliado apenas por se tratar de um procedimento minimamente invasivo. Nos poucos casos em que a ressecção é necessária para tratar tumores hepáticos benignos, a ressecção hepática laparoscópica deve ser o método preferido.

\section{REFERÊNCIAS}

1. Arnaoutakis DJ, Kim $Y$, Pulitano $C$, Zaydfudim $V$, Squires $M H$, Kooby D, Groeschl R, Alexandrescu S, Bauer TW, Bloomston M, et al. Management of biliary cystic tumors: a multi-institutional analysis of a rare liver tumor. Ann Surg. 2015;261(2):361-7. doi: 10.1097/SLA.0000000000000543.

2. Assis BS, Coelho FF, Jeismann VB, KrugerJAP, Fonseca GM, Cecconello I, Herman P. Total laparoscopic vs. open liver resection: comparative study with propensity score matching analysis. Arq Bras Cir Dig. 2020;33(1):e1494. doi: 10.1590/0102-672020190001e1494.

3. Bioulac-Sage P, Laumonier H, Couchy G, Le Bail B, Sa Cunha A, Rullier A, Laurent C, Blanc JF, Cubel G, Trillaud H, et al. Hepatocellular adenoma managementand phenotypic classification: the Bordeaux experience. Hepatology.2009;50(2):481-9. doi:10.1002/hep.22995.

4. Calame P, Tyrode G, Weil Verhoeven D, Félix S, Klompenhouwer AJ, Di Martino V, Delabrousse E, Thévenot T. Clinical characteristics and outcomes of patients with hepatic angiomyolipoma: A literature review. World J Gastroenterol. 2021;27(19):2299-2311. doi: 10.3748/wjg.v27.i19.2299.

5. Cipriani F, Alzoubi M, Fuks D, Ratti F, Kawai T, Berardi G, Barkhatov L, Lainas P, Van der Poel M, Faoury M, et al. Pure laparoscopic versus open hemihepatectomy: a critical assessment and realistic expectations - a propensity score-based analysis of right and left hemihepatectomies from nine European tertiary referral centers. J Hepatobiliary Pancreat Sci. 2020;27(1):3-15. doi: 10.1002/jhbp.662.

6. Ciria R, Cherqui D, GellerDA, BricenoJ, Wakabayashi G. Comparative Short-term Benefits of Laparoscopic Liver Resection: 9000 Cases and Climbing. Ann Surg. 2016;263(4):761-77. doi: 10.1097/ SLA.0000000000001413.

7. Coelho FF, Kruger JA, Fonseca GM, Araújo RL, Jeismann VB, Perini MV, Lupinacci RM, Cecconello I, Herman P. Laparoscopic liver resection: Experience based guidelines. World J Gastrointest Surg. 2016;8(1):5-26. doi: 10.4240/wjgs.v8.i1.5.

8. Descottes B, Glineur D, Lachachi F, Valleix D, Paineau J, Hamy A, Morino M, Bismuth H, Castaing D, Savier E, et al. Laparoscopic liver resection of benign liver tumors. Surg Endosc. 2003;17(1):23-30. doi: 10.1007/s00464-002-9047-8.

9. Dindo D, Demartines N, Clavien PA. Classification of surgical complications: a new proposal with evaluation in a cohort of 6336 patients and results of a survey. Ann Surg. 2004;240(2):205-13. doi: 10.1097/01.sla.0000133083.54934.ae.

10. Duarte VC, Coelho FF, Valverde A, Danoussou D, Kruger JAP, Zuber K, Fonseca GM, Jeismann VB, Herman P, Lupinacci RM. Minimally invasive versus open right hepatectomy: comparative study with propensity score matching analysis. BMC Surg. 2020;20(1):260. doi: 10.1186/s12893-020-00919-0. 
11. Erdogan D, Kloek J, Lamers WH, Offerhaus GJ, Busch OR, Gouma DJ, van Gulik TM. Mucinous cystadenomas in liver: management and origin. Dig Surg. 2010;27(1):19-23. doi: 10.1159/000268110.

12. Farges $O$, Ferreira N, Dokmak S, Belghiti J, Bedossa P, Paradis V. Changing trends in malignant transformation of hepatocellular adenoma. Gut. 2011;60(1):85-9. doi: 10.1136/gut.2010.222109.

13. Herman $P$, Coelho FF, Perini MV, Lupinacci RM, D'Albuquerque LA, Cecconello I. Hepatocellular adenoma: an excellent indication for laparoscopic liver resection. HPB (Oxford). 2012;14(6):390-5. doi: 10.1111/j.1477-2574.2012.00463.x.

14. Herman P, Fonseca GM, Kruger JAP, Jeismann VB, Coelho FF. Guidelines for the Treatment of HepatocellularAdenoma in the Era of Molecular Biology: An Experience-Based Surgeons' Perspective. J Gastrointest Surg. 2021;25(6):1494-1502. doi: 10.1007/s11605020-04724-1.

15. Herman $P$, Pugliese $V$, Machado $M A$, Montagnini $A L$, Salem $M Z$, Bacchella T, D'Albuquerque LA, Saad WA, Machado MC, Pinotti HW. Hepatic adenoma and focal nodular hyperplasia: differential diagnosis and treatment. World J Surg. 2000;24(3):372-6. doi: $10.1007 /$ s002689910059.

16. Herman $P$, Costa $M L$, Machado MA, Pugliese $V$, D'Albuquerque LA, Machado MC, Gama-Rodrigues JJ, Saad WA. Management of hepatic hemangiomas: a 14-year experience. J Gastrointest Surg. 2005;9(6):853-9. doi: 10.1016/j.gassur.2005.01.292.

17. Hsee LC, McCall JL, Koea JB. Focal nodular hyperplasia: what are the indications for resection? HPB (Oxford). 2005;7(4):298-302. doi: 10.1080/13651820500273624.

18. Kanji ZS, Rocha FG. Premalignant Lesions of the Biliary Tract. Surg Clin North Am. 2019;99(2):301-314. doi:10.1016/j.suc.2018.11.007.

19. Klompenhouwer AJ, Verver D, Janki S, Bramer WM, Doukas M, Dwarkasing RS, de Man RA, IJzermans JNM. Management of hepatic angiomyolipoma: A systematic review. Liver Int. 2017;37(9):12721280. doi: 10.1111/liv.13381.

20. Laurent A, Dokmak S, Nault JC, Pruvot FR, Fabre JM, Letoublon C, Bachellier P, Capussotti L, Farges O, Mabrut JY, et al. European experience of 573 liver resections for hepatocellular adenoma: a cross-sectional study by the AFC-HCA-2013 study group. HPB (Oxford). 2016;18(9):748-55. doi: 10.1016/j.hpb.2016.06.011.

21. Miller GC, Campbell CM, Manoharan B, Bryant R, Cavallucci D, O'Rourke N, Clouston AD. Subclassification of hepatocellular adenomas: practical considerations in the implementation of the
Bordeaux criteria. Pathology. 2018;50(6):593-599. doi: 10.1016/j. pathol.2018.05.003.

22. Nault JC, Couchy G, Balabaud C, Morcrette G, Caruso S, Blanc JF, Bacq Y, CalderaroJ, Paradis V, Ramos J, etal. MolecularClassification of Hepatocellular Adenoma Associates With Risk Factors, Bleeding, and Malignant Transformation. Gastroenterology.2017;152(4):880894.e6. doi: 10.1053/j.gastro.2016.11.042.

23. Nault JC, Bioulac-Sage P, Zucman-Rossi J. Hepatocellular benign tumors-from molecular classification to personalized clinical care. Gastroenterology. 2013;144(5):888-902. doi: 10.1053/j. gastro.2013.02.032.

24. Nguyen KT, Gamblin TC, Geller DA. World review of laparoscopic liver resection-2,804 patients. Ann Surg. 2009;250(5):831-41. doi: 10.1097/SLA.0b013e3181b0c4df.

25. PekoljJ, Clariá SánchezR, Salceda J, Maurette RJ, Schelotto PB, Pierini L, Cánepa E, Moro M, Stork G, Resio N, et al. Laparoscopic Liver Resection: A South American Experience with 2887 Cases. World J Surg. 2020;44(11):3868-3874. doi: 10.1007/s00268-020-05646-4.

26. Seow J, McGill M, Wang W, Smith P, Goodwin M. Imaging hepatic angiomyolipomas: key features and avoiding errors. Clin Radiol. 2020;75(2):88-99. doi: 10.1016/j.crad.2019.09.135.

27. Simo KA, Mckillop IH, Ahrens WA, Martinie JB, lannitti DA, Sindram D. Invasive biliary mucinous cystic neoplasm: a review. HPB (Oxford). 2012;14(11):725-40. doi: 10.1111/j.1477-2574.2012.00532.x.

28. Toro A, Gagner M, Di Carlo I. Has laparoscopy increased surgical indications for benign tumors of the liver? Langenbecks Arch Surg. 2013;398(2):195-210. doi: 10.1007/s00423-012-1012-y.

29. van Aalten SM, Thomeer MG, Terkivatan T, Dwarkasing RS, Verheij J, de Man RA, ljzermans JN. Hepatocellular adenomas: correlation of MR imaging findings with pathologic subtype classification. Radiology. 2011;261(1):172-81. doi: 10.1148/radiol.11110023.

30. Wabitsch S, Kästner A, Haber PK, Benzing C, Krenzien F, Andreou A, Kamali C, Lenz K, Pratschke J, Schmelzle M. Laparoscopic Versus Open Liver Resection for Benign Tumors and Lesions: A Case Matched Study with Propensity Score Matching.J LaparoendoscAdv Surg Tech A. 2019;29(12):1518-1525. doi: 10.1089/lap.2019.0427.

31. Wakabayashi G, Cherqui D, Geller DA, Han HS, Kaneko H, Buell JF. Laparoscopic hepatectomy is theoretically better than open hepatectomy: preparing for the 2nd International Consensus Conference on Laparoscopic Liver Resection. J Hepatobiliary Pancreat Sci. 2014;21(10):723-31. doi: 10.1002/jhbp.139. 